\title{
Assessment on Tokamak Fusion Power Plant to Contribute to Global Climate Stabilization in the Framework of Paris Agreement
}

\author{
Ryoji HIWATARI, Takuya GOTO ${ }^{1)}$ and Joint Special Design Team for Fusion DEMO \\ National Institute for Quantum and Radiological Science and Technology, Rokkasho, Aomori 039-3212, Japan \\ ${ }^{1)}$ National Institute for Fusion Science, National Institutes of Natural Sciences, Toki, Gifu 509-5292, Japan
}

(Received 27 July 2018 / Accepted 9 January 2019)

\begin{abstract}
A cost model for a tokamak fusion power plant (FPP) is improved to evaluate material cost and manufacture cost, separately. Then, the improved cost model is applied to a commercial tokamak FPP, and reduction of FPP construction cost is investigated considering learning effect on manufacture of the fusion island part and the advanced manufacture of toroidal field (TF) coils. Finally, a development scenario of a tokamak FPP is proposed to contribute substantially to global climate stabilization under the framework of the Paris Agreement.
\end{abstract}

(C) 2019 The Japan Society of Plasma Science and Nuclear Fusion Research

Keywords: global climate change, fusion energy, construction cost, cost model, manufacture cost, long term world energy scenario

DOI: $10.1585 /$ pfr. 14.1305047

Fusion energy has been considered as one of the advanced technologies to stabilize the global climate change [1]. Recently, Paris Agreement [2] aims at holding increase in the global average temperature to well below $2{ }^{\circ} \mathrm{C}$ above pre-industrial levels, and also aims at pursuing efforts to limit the temperature increase to $1.5^{\circ} \mathrm{C}$ above pre-industrial levels. It is recognized that those targets would significantly reduce the risks and impacts of climate change.

A latest analysis on a long-term world energy scenario considering Paris Agreement reveals that there is an introduction condition of fusion energy into the energy market under scenarios of low greenhouse gas emission [3]. In case of an energy scenario to hold the temperature increase less than $2^{\circ} \mathrm{C}$ in 2100 , an introduction condition of fusion energy has been evaluated as a breakeven price of construction unit costs of 2 - 8 dollar/W under the condition of plant availability $90 \%$. Here, the unit cost is defined by the net electric power output.

This paper promptly reports the initial result of economic assessment on tokamak fusion power plant (FPP) to discuss its possibility to contribute to Paris Agreement's global climate stabilization.

Conceptual design studies about a commercial fusion plant have been carried out up to now. As for magnetic fusion reactor concepts, large-scale conceptual power plant designs have been carried out [4]. Representative recent commercial power plant concepts are considered as CREST in Japan [5], ARIES ACT-series in US [6], and PPCS in EU [7]. Among those concepts, CREST is the most compact tokamak (i.e. major radius $5.4 \mathrm{~m}$ and aspect ratio 3.4). As for an initial assessment considering Paris Agreement, CREST is considered as a typical commercial

author's e-mail: hiwatari.ryoji@qst.go.jp
FPP candidate in this paper. Major parameters of CREST are as follows: net electric power $1.16 \mathrm{GW}$, fusion power $2.97 \mathrm{GW}$, max. toroidal magnetic field $12.5 \mathrm{~T}$, plasma current $12 \mathrm{MA}$ [5].

Recently, a cost analysis model is being improved based on the previous cost model $[8,9]$, considering the ITER experience, detailed treatment of power plant devices/buildings, updates of material unit cost, and so on. Primary improvement is separation of material cost and manufacturing cost as for fusion island (which means devices around a fusion reactor core). Material cost $C_{F I_{-} X}^{m a t}$ dollar and manufacture one $C_{F I-X}^{m a n}$ dollar of fusion island components are estimated by:

$$
\begin{aligned}
& C_{F I_{-} X}^{\text {mat }}=\rho_{F I_{-} X} \times V_{F I_{-} X} \times \$_{F I_{-} X}, \\
& C_{F I_{-} X}^{\text {man }}=C_{F I_{-} X}^{r e f} \times\left(M_{F I_{-} X} / M_{F I_{-} X}^{r e f}\right)^{\gamma_{\text {man }}},
\end{aligned}
$$

where $\rho_{F I \_X} \mathrm{~kg} / \mathrm{m}^{3}, V_{F I \_X} \mathrm{~m}^{3}, \$_{F I_{-} X}$ dollar $/ \mathrm{kg}, C_{F I_{-} X}^{r e f}$ dollar, $M_{F I \_X} \mathrm{~kg}, M_{F I_{-} X}^{r e f} \mathrm{~kg}, \gamma_{\operatorname{man}}$ are material mass density of component X, volume of component X, unit cost, reference manufacture cost of component $\mathrm{X}$, mass of component $X$, reference mass of component $X$ and scaling factor $\gamma_{\text {man }}$, respectively. Now, scaling factor $\gamma_{\text {man }}$ is assumed to be 0.6 [10]. Another major improvement is detailed consideration of all devices and buildings required in an FPP such as a hot cell, a PF coil winding building, a tritium plant, a cryogenic system, a power supply system, and so on. Those points were not clearly included in the previous cost model [9], which had evaluated construction cost at about 5 billion dollars for CREST [11]. Several reference values in Eqs. (1) and (2) are derived from ITER-FDR cost assessment conducted by JAERI [12], and detailed implementation into the improved cost model will be submitted as another paper.

The above improved cost model is applied to the 
CREST concept and it reveals increase of construction cost of CREST from about 5 (which was evaluated by the previous cost model [9]) to about 9.3 billion dollars, because additional devices and buildings such as the assembly/maintenance system, the hot cell, the PF winding building, the tritium plant, the power supply system, the cryogenic system, are counted, and recent material cost increases such as beryllium for neutron multiplier $(0.17 \mathrm{mil}-$ lion dollars/ton in 2001, 0.49 million dollars/ton in 2015 [13]).

Figure 1 shows the breakdown of the construction cost by the improved cost model. It consists of the fusion island part (38\%), devices (41\%) and buildings (21\%). The fusion island part is separated mainly into the superconducting (SC) coil, the breeding blanket, the shield blanket, the divertor, and the vacuum vessel/thermal shield etc., and each component of the fusion island are evaluated from the viewpoint of material and manufacture costs. For the SC coils, the material cost and manufacture one are evaluated at 473 million dollars and 1331 million dollars, respectively. The cost of the SC coils is corresponding to $19 \%$ of the total construction cost, but it amounts up to $51 \%$ of the fusion island cost. The material cost of the breeding blanket is larger than its manufacture one, because the material cost includes the pebble production of tritium breeder and neutron multiplier.

The devices part consists of the turbine system, the heat transfer one, the heating one, the power supply one, the cryogenic one, the vacuum pump one, the fueling one, the diagnostic one, the CODAC (COntrol \& Data Acquisition, Communication) one, the tritium plant one, the hot cell facilities, the assembly and maintenance one. Almost all devices required for an FPP are considered, whereas the previous model considers them as a representative component such as balance of plant (BOP) $[8,9]$. This is an improved point from the previous model.
The building part consists of the reactor building, the hot cell one, the heating one, the emergency power supply one, the magnet power supply one, the cryo-plant one, the turbine one, the PF winding one, etc. The major building required for a tokamak FPP is considered, whereas the previous model considers them as several representative buildings $[8,9]$. This point is also an improved point from the previous model. Such detailed consideration on devices and buildings is one of the major reasons for increase of the estimated construction cost from the previous model to the present improved model.

Basic concept to evaluate each cost above devices and buildings is derived from scaling law with the reference parameter from the ITER-FDR cost analysis and the present power station experience such as a light water reactor and a thermal power station. However, there are still some issues on the present cost model to be modified. For example, the cost of the heating device is evaluated at small value of $1 \%$, which comes from the large bootstrap fraction over $90 \%$ and small additional heating power during the rating operation. The ramp-up phase including LH transition should be considered to evaluate a reasonable heating power and its device cost. And decommissioning cost is not included yet. Such detailed consideration and modification will be discussed in another paper.

The resultant construction unit cost is estimated as 7.98 dollar/W for the first commercial FPP, which is the upper limit of a breakeven price of construction unit cost. In this case, introduction area of fusion energy would be not worldwide but very limited based on [3], and contribution of fusion energy to Paris Agreement's global climate stabilization would be small, according to the latest world energy scenario analysis [3].

This construction cost shown in Fig. 1 is considered as that of the first commercial FPP based on the ITER and the present power station experience, and advancement and

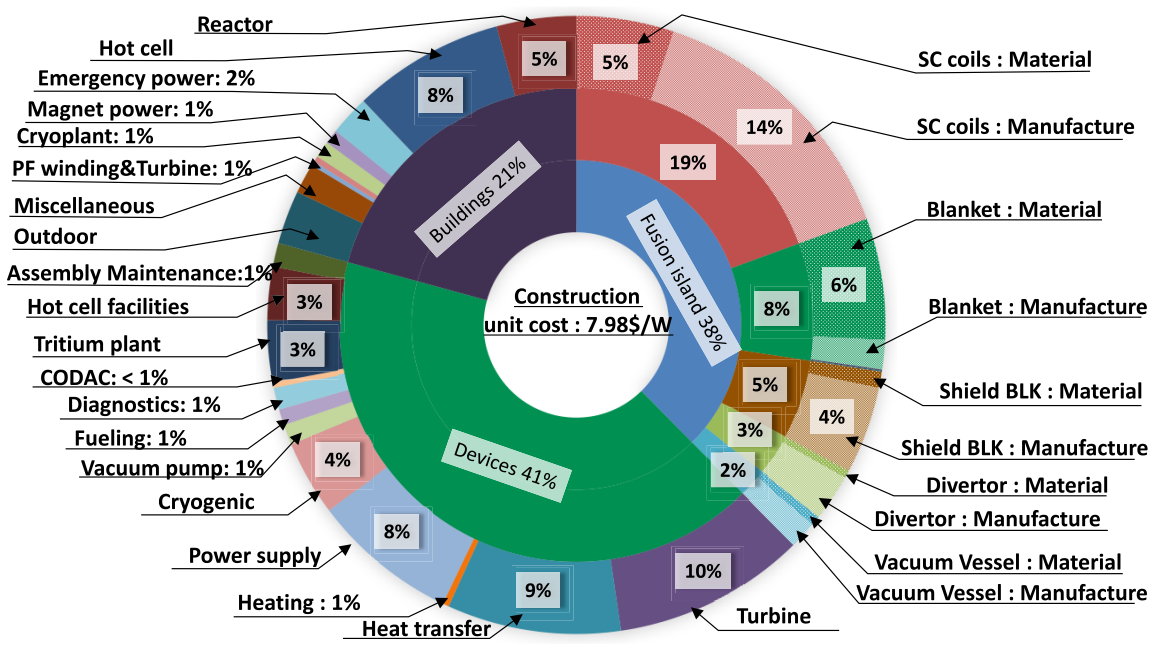

Fig. 1 Construction unit cost of the first CREST and its detailed breakdown and percentage. 
learning effect for manufacture technique are not considered yet. Considering learning effect, the construction cost of the following commercial FPP would be expected to reduce. Hence, 10th kind of a commercial FPP will be discussed for contribution to the global climate stabilization under the framework of Paris Agreement.

Moreover, advancement of manufacture is another effect on cost reduction. For example, advancement of winding method of a toroidal field (TF) coil is being explored in $\mathrm{EU}$, where the rectangular SC conductor is directly winded without radial plate applied in ITER TF coils. The expected cost reduction of TF coil by a new winding method is evaluated at $50 \%$ [14].

To forecast changes in technology cost, the learning curve is widely applied as follow,

$$
Y=a X^{b}
$$

where $Y$ is the unit cost of the technology and $X$ represents cumulative manufacture experience. The constants $a$ and $b$ in Eq. (3) represent the unit cost of the first unit and the rate of cost reduction, respectively. The fractional reduction in cost associated with a doubling of manufacture experience is referred to as the learning rate (LR) and is given by

$$
L R=1-2^{b} .
$$

The recent mean $L R$ for the electric supply technology is evaluated at $8.3 \%-15 \%$ for coal and natural gas power plant, whereas the $L R$ of nuclear power plant is evaluated at from negative to $6 \%$ [15]. The reason of the negative $L R$ of nuclear power plant is caused not from technological issues, but from regulation strengthening. Here, we apply $L R=15 \%(b \sim-0.235)$, which is the upper value of $L R$ for coal and natural gas power plants, for manufacture cost of a commercial tokamak FPP under the condition that regulation wouldn't change. We expect that $L R$ for manufacture cost for a tokamak FPP would be as high as $15 \%$ of the coal and natural gas power plant, because the coal and natural gas power plant is considered as well matured technology, whereas the tokamak FPP is done as a new one and the effect of learning experience for a tokamak FPP is supposed to be larger than the coal and natural gas power plant.

We apply $L R=15 \%$ to manufacture cost of the fusion island part, and we also expect $50 \%$ cost reduction of TF coils by advancement of manufacture according to EU proposal [14]. Figure 2 shows the breakdown of construction cost of the 10th kind of a commercial tokamak FPP. Fraction of the fusion island part decreases from $38 \%$ to $28 \%$. Here, we assume that the cost of devices and buildings doesn't conservatively change so much from the first commercial tokamak FPP, because most of devises and buildings are considered to be insensitive to learning effect. The construction unit cost is estimated as 6.87 (dollar/W) for 10th commercial tokamak FPP. According to the latest analysis on a long-term world energy scenario considering Paris Agreement [3], that construction unit cost 6.87 dollar/W enables introduction of fusion energy into China, EU, India, Japan and Korea. Introduction condition into US and Russia is more severe than 6.87 dollar/W, because of their large energy self-sufficiency. However, the substantial introduction of fusion energy into the world energy market could be realized under such construction unit cost. That means that fusion energy can be a substantial candidate in the energy source to contribute to global climate stabilization under the framework of the Paris Agreement.

Finally, the technological issues are also briefly assessed for the substantial contribution of an FPP to global climate stabilization, and the results mentioned above are summarized as a development scenario of a tokamak FPP.

As for a core plasma design, the reversed shear configuration with the normalized beta value $\beta_{N} \sim 5.5$ is applied

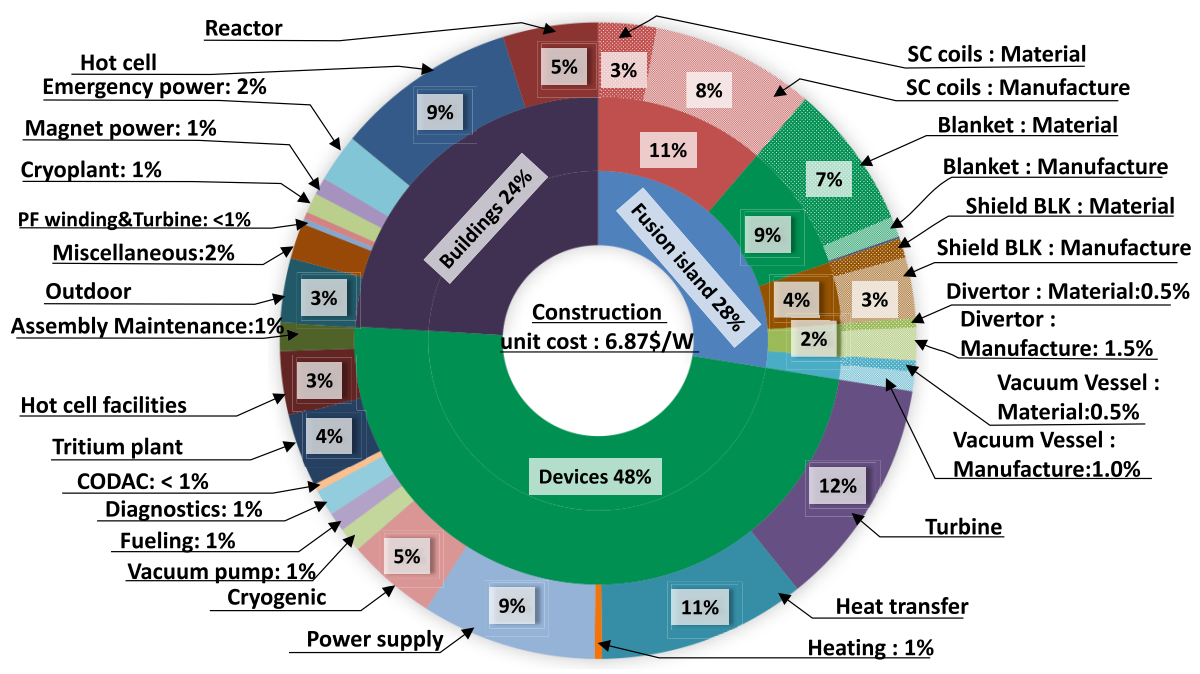

Fig. 2 Construction unit cost of 10th CREST and its detailed breakdown and percentage, considering learning effect on manufacture of the fusion island and manufacture advancement of TF coils. 


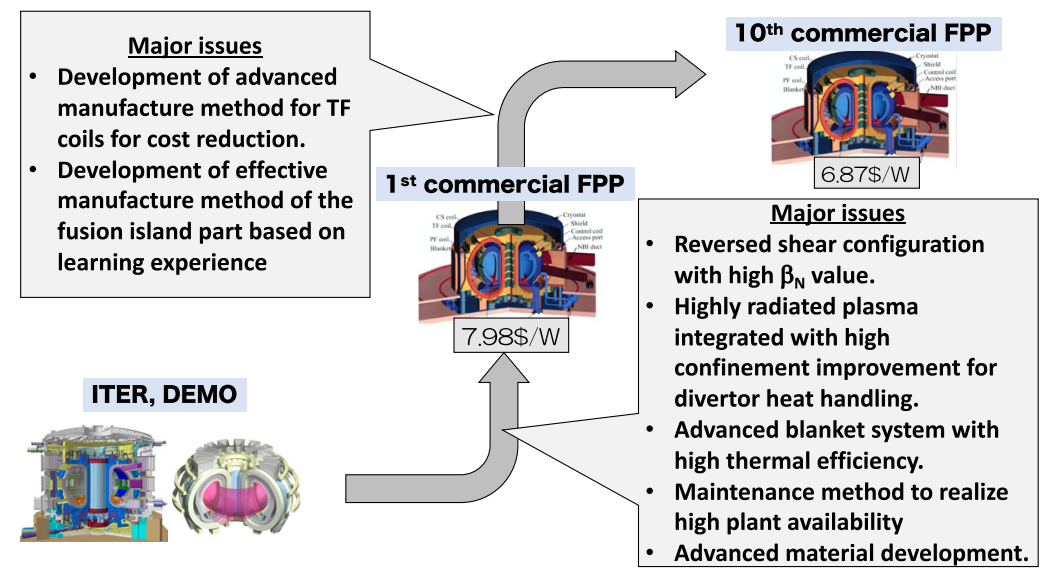

Fig. 3 Development scenario of a tokamak FPP and technological issues to contribute to global climate stabilization under the framework of Paris Agreement.

to achieve the net electric power $1.16 \mathrm{GW}$ with a compact device size of $5.4 \mathrm{~m}$, and such high-performance plasma is beyond the ITER project. However, the target of JT-60SA includes up to such high $\beta_{N}$ considering commercialization of FPP [16], and demonstration of such high-performance plasma has to be pursued. Divertor heat handling is another critical issue. In the CREST concept, impurity radiation not only from the SOL-divertor region but also from core plasma region is required in order to keep the divertor heat load less than $10 \mathrm{MW} / \mathrm{m}^{2}$ similar to that of ITER [5]. Such highly radiated core plasma operation, which is proposed in the EU demonstration reactor (DEMO) physics [17], requires high confinement improvement, and such integrated plasma performance has to be demonstrated in ITER, JT60SA or DEMOs.

Major engineering advancements from ITER or DEMO to CREST are high thermal efficiency close to $40 \%$, maintenance method realizing high plant availability close to $90 \%$, and material development. The specification of TF coil such as max. field (12.5 T) for CREST is almost similar to that of ITER. Generally speaking, high thermal efficiency requires high coolant temperature. In the case of water coolant, a pressurized water condition applied to the Japanese ITER-TBM (Test Blanket Module) plan is not enough to achieve thermal efficiency close to $40 \%$. Basically, super-heated or super-critical condition of the blanket coolant is required in case of water coolant $[5,18]$. Of course, coolant choice is not restricted to water, and a different concept proposed for ITER-TBMs should be considered. Anyway, the advanced blanket system has to be developed using DEMO-TBM as proposed in Japan [19]. Correspondingly, materials applied to such advanced blanket have to be developed.

Figure 3 shows a development scenario of a commercial tokamak FPP and technological issues to contribute to global climate stabilization under the framework of Paris Agreement. From ITER and DEMO to 1st commercial
FPP, several physical and engineering issues mentioned above have to be resolved. At this stage of 1 st commercial FPP, the construction unit cost achieves to the upper limit of a breakeven price 8.0 dollar/W for introduction into the worldwide energy scenario [3]. Considering learning effect on manufacture of fusion island and advancement of manufacture of TF coil, the construction unit cost of 10th kind of a commercial FPP is expected to reduce down to 6.87 dollar/W. This construction unit cost enables fusion energy to contribute substantially to global climate stabilization under the framework of the Paris Agreement, according to the latest energy scenario [3].

In summary, we have improved the cost model for a tokamak FPP. The improved cost model enables to evaluate material cost and manufacture one, separately. Then, we have applied the improved cost model to a typical commercial tokamak FPP, and have evaluated construction cost reduction by considering learning effect on manufacture of the fusion island part and the advanced manufacture of TF coils. Finally, we have proposed a development scenario of a commercial tokamak FPP to contribute substantially to global climate stabilization under the framework of the Paris Agreement.

This study has been supported by a grant-in-aid for Joint Special Design Team for Fusion DEMO from the Ministry of Education, Culture, Sports, Science and Technology.

[1] M.I. Hoffert et al., Science 298, Issue 5595, 981 (2002).

[2] Paris agreement, Framework Convention on Climate Changes, United Nations (2016). http://unfccc.int/paris_agreement/items/9485.php

[3] K. Gi et al., "Assessment of Potential and Breakeven Prices of Fusion Power Plants under Low-Carbon Development Scenarios”, SEE/P7-1, 26th IAEA Fusion Energy Conference, Kyoto Japan, (2016). https://nucleus.iaea.org/sites/ fusionportal/Shared\%20Documents/FEC\%202016/ fec2016-preprints/preprint0084.pdf 
[4] L.A. El-Guebaly, Energies 3, 1067 (2010).

[5] K. Okano et al., Nucl. Fusion 40, 635 (2000).

[6] C.E. Kessel et al., Fusion Sci Technol. 67, 1 (2015).

[7] Final Report of the European Fusion Power Plant Conceptual Study (PPCS), EFDA-RP-RE-5.0, EUROPEAN FUSION DEVELOPMENT AGREEMENT (2005).

[8] R. Hiwatari et al., J. Plasma Fusion. Res. 81, 903 (2005).

[9] T. Yoshida et al., "Development of Cost Assessment Code of Fusion Power Reactors", T94001, CRIEPI research report, Central Research Institute of Electric Power Industry (CRIEPI), 1994 (in Japanese).

[10] M.A. Green and B.P. Strauss, IEEE Trans. Appl. Supercond. 18, No. 2, LBNL-63482 (2007).

[11] Y. Ogawa et al., Socio-Economic Research for Innovative Energy Technologies, NIFS-MEMO-049, NIFS (2006).
[12] ITER-FDR cost estimation report conducted by JAERI (1998).

[13] Beryllium, Supply-Demand Statistics, Historical Statistics for Mineral and Material Commodities in the United States, U.S. Geological Survey Data Series 140, https://minerals. usgs.gov/minerals/pubs/historical-statistics/

[14] P. Bruzzone et al., IEEE Trans. Appl. Supercond. 24, No. 3, 4201504 (2014).

[15] E.S. Rubin et al., Energy Policy 86, 198 (2015).

[16] JT-60SA Research Plan, version 3.3 http://www.jt60sa.org/ pdfs/JT-60SA_Res_Plan.pdf

[17] R. Wenninger et al., Nucl. Fusion 55, 063003 (2015).

[18] R. Hiwatari et al., Nucl. Fusion 45, 96 (2005).

[19] H. Yamada et al., Fusion Eng. Des. 109-111, 1318 (2016). 